TITLE:

\title{
Spatiotemporal behaviors of excited Xe atoms in unit discharge cell of ac-type plasma display panel studied by laser spectroscopic microscopy
}

\author{
AUTHOR(S): \\ Tachibana, K; Feng, SJ; Sakai, T
}

\section{CITATION:}

Tachibana, K ... [et al]. Spatiotemporal behaviors of excited Xe atoms in unit discharge cell of ac-type plasma display panel studied by laser spectroscopic microscopy. JOURNAL OF APPLIED PHYSICS 2000, 88(9): 4967-4974

\section{ISSUE DATE:}

2000-11-01

URL:

http://hdl.handle.net/2433/50088

\section{RIGHT:}

Copyright 2000 American Institute of Physics. This article may be downloaded for personal use only. Any other use requires prior permission of the author and the American Institute of Physics. 


\title{
Spatiotemporal behaviors of excited Xe atoms in unit discharge cell of ac- type plasma display panel studied by laser spectroscopic microscopy
}

\author{
Kunihide Tachibana ${ }^{a)}$ and Shaojun Feng \\ Department of Electronic Science and Engineering, Kyoto University, Yoshida-honmachi Sakyo-ku, \\ Kyoto 606-8501, Japan \\ Tetsuo Sakaib) \\ Hyundai Electronics Japan Co., Ltd., KSP Bldg. R\&D-D937, 3-2-1 Sakado, Takatsu-ku, \\ Kawasaki 213-0012, Japan
}

(Received 25 February 2000; accepted for publication 7 August 2000)

Two-dimensional spatiotemporal behaviors of excited Xe atoms in the $1 s_{4}$ resonance state and the $1 s_{5}$ metastable state were measured in a unit discharge cell of an ac-type plasma display panel by a laser absorption technique combined with an optical microscope. The measured density of $\mathrm{Xe}\left(1 s_{5}\right)$ has two large peaks on both the temporal anode and cathode sides. The peak at the anode has a narrower spatial distribution while the peak at the cathode is distributed over the electrode area. In its temporal behavior, the anode peak rises slightly faster than the peak at the cathode and decays faster at the beginning of afterglow, but both peaks tend to have the same decay rate in the later period. The behavior of $\mathrm{Xe}\left(1 s_{4}\right)$ shows similar features, but the decay rate is much larger, corresponding to the effective lifetime of imprisoned resonance radiation. The maximum densities of $\mathrm{Xe}\left(1 s_{5}\right)$ and $\mathrm{Xe}\left(1 s_{4}\right)$ are $5 \times 10^{13}$ and $2 \times 10^{13} \mathrm{~cm}^{-3}$, respectively. Emission from $\mathrm{Xe}(2 p)$ atoms was also observed, and this nearly followed the current wave form. With these results, we estimated the efficiency of vacuum ultraviolet emissions from excited $\mathrm{Xe}\left(1 s_{4}\right)$ atoms and $\mathrm{Xe}_{2}^{*}$ excimers formed from $\mathrm{Xe}\left(1 s_{5}\right)$ atoms. (C) 2000 American Institute of Physics.

[S0021-8979(00)08221-9]

\section{INTRODUCTION}

Recently, major developments in the technology of plasma display panels (PDPs) permitted the production of large-area wall-mountable displays for high-definition television receivers. ${ }^{1}$ In general, there are two types of PDPs, i.e., dc type and ac type. In both types, the vacuum ultraviolet (VUV) emissions from excited $\mathrm{Xe}^{*}$ atoms and $\mathrm{Xe}_{2}^{*}$ dimers in Xe plasmas diluted with $\mathrm{He}$ or $\mathrm{Ne}$ are converted into visible red, green, and blue dots by using the appropriate phosphors. At the moment, it is commonly recognized that the ac type is advantageous to the dc type in luminous efficiency and in tractability for manufacturing large panels due to its simpler structure. ${ }^{2}$ Thus, the ac type has been practically adopted in commercial mass production. However, no quantitative estimation of plasma characteristics in a unit discharge cell of the ac-type PDP has been carried out aside from some qualitative measurements of VUV emissions, ${ }^{3,4}$ although a few theoretical approaches have been investigated by using fluid and particle models. ${ }^{5-8}$

In this work, we constructed a coplanar ac-type test panel filled with a mixture of $\mathrm{Xe}$ and $\mathrm{Ne}$, and measured the spatiotemporal behaviors of the absolute densities of excited $\mathrm{Xe}$ atoms in the $1 s_{4}$ and $1 s_{5}$ (in Paschen notation) levels. From the measurements, we quantitatively estimated the behaviors of the line emission at $147 \mathrm{~nm}$ from the $\mathrm{Xe}\left(1 s_{4}\right)$

\footnotetext{
${ }^{a)}$ Electronic-mail: tatibana@kuee.kyoto-u.ac.jp

${ }^{b}$ Present address: Display Research Laboratories Co. Ltd., Tamagawadai, Setagaya-ku, Tokyo 158-0096, Japan.
}

atoms and the band emission at $173 \mathrm{~nm}$ from the $\mathrm{Xe}_{2}^{*}$ excimers formed by the three body collisions of $\mathrm{Xe}\left(1 s_{5}\right)$ atoms. A diode laser absorption method combined with an optical microscope was used for the measurement, as in the case of the dc-type cell reported previously. ${ }^{9}$ The cell structure and operation conditions are described in Sec. II along with the experimental setup and procedure. The results of spatiotemporal densities of excited Xe atoms are given in Sec. III with a discussion on the efficiency of VUV emissions derived from the data as well as the measured current wave forms. Characteristics of these spatiotemporal behaviors of the actype cell is also discussed in a comparison with that of a dc-type cell.

\section{EXPERIMENTAL}

\section{A. Setup and procedure}

The cell structure of a test panel is shown schematically in Fig. $1 .{ }^{10}$ As seen from Fig. 1, it has a coplanar structure with a pair of sustain electrodes on the front glass plate made of transparent indium-tin-oxide (ITO) films backed up with metallic bus electrodes. The ITO electrodes are covered successively by a dielectric layer of $20 \mu \mathrm{m}$ thickness and an $\mathrm{MgO}$ film of $0.5 \mu \mathrm{m}$ thickness. The front plate is separated from the back glass plate by $130 \mu \mathrm{m}$ with ribs made of insulating ceramics. As opposed to a conventional cell structure, this test panel has no addressing electrode or phosphor coating to allow access by the probe laser beam. A mixture of $\mathrm{Xe}(10 \%)$ with $\mathrm{Ne}$ was filled in the panel at a pressure of 350 Torr. Square wave pulses of $2 \mu$ s duration were applied 


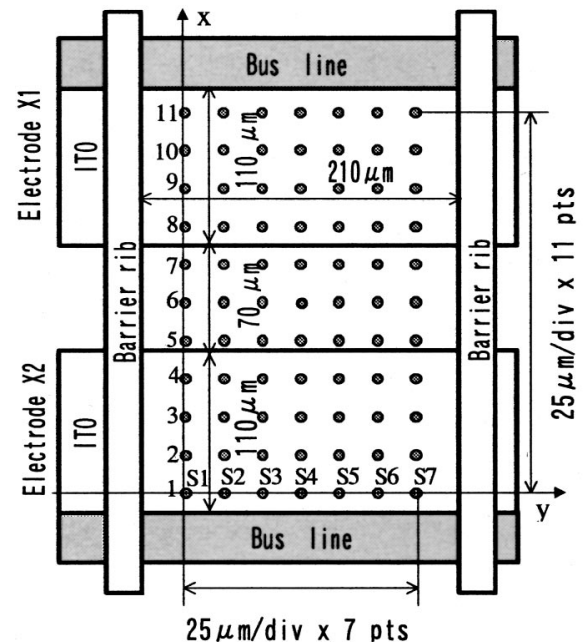

(a)

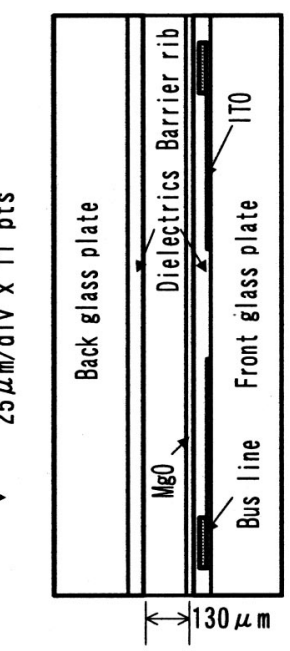

(b)
FIG. 1. Schematic of unit cell structure of test panel: (a) front view and (b) side view. Measuring points are assigned by a matrix of numbered $x-y$ coordinates.

to the electrode $\mathrm{X} 1$ with a period of $12 \mu \mathrm{s}$, and the complementary pulses delayed by $6 \mu$ s were applied to X2. Therefore, the polarity of the differential voltage applied between these electrodes effectively reverses every $6 \mu \mathrm{s}$. The peak value of the applied pulse voltage $V_{s}$ was changed from 190 to $220 \mathrm{~V}$, but a typical value of $200 \mathrm{~V}$ was used in most of the measurements.

The setup of spectroscopic microscopy shown in Fig. 2 is the same as that used previously. ${ }^{9}$ In this work, however, we used a commercial diode laser system, of which the wavelength can be tuned or scanned by the angle of the end tuning mirror of an external grazing incidence (Littman/ Metcalf-type) cavity. The tuning mirror was driven by a motor for coarse movements or by a piezo actuator for fine movements. In the measurements of spatiotemporal behavior of excited Xe atom density, $823.1 \mathrm{~nm}\left(2 p_{6}-1 s_{5}\right)$ and 828.0 $\mathrm{nm}\left(2 p_{5}-1 s_{4}\right)$ lines were used for the $1 s_{5}$ metastable level and the $1 s_{4}$ resonance level, respectively. The diode laser beam of a few milliwatts power level was divided into two parts by a beam splitter. One beam was used to tune the

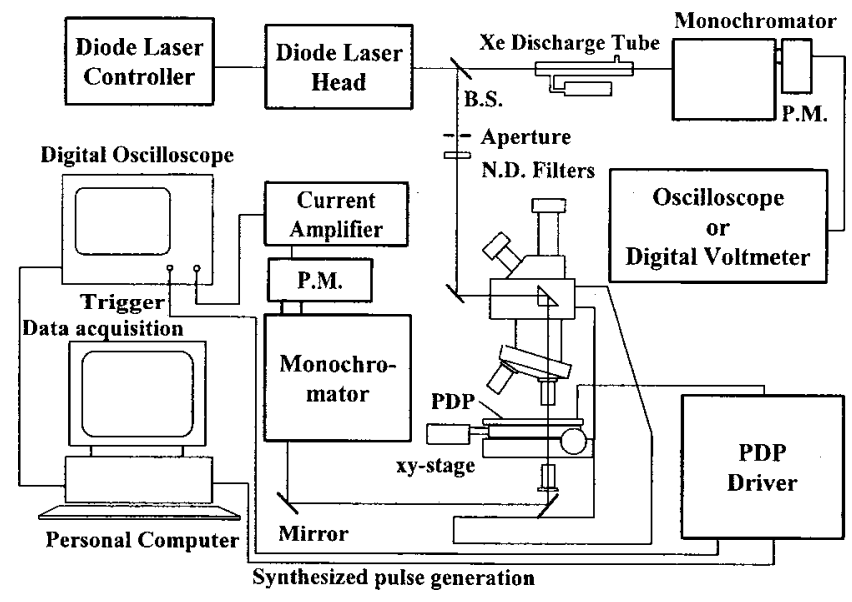

FIG. 2. Schematic of experimental setup for laser spectroscopic microscopy. wavelength to the center of the respective absorption line of $\mathrm{Xe}$ atoms by using a reference Xe discharge tube filled with pure Xe gas at 2 Torr, and the other beam was led into an optical microscope on which the test panel was mounted. The laser beam intensity was attenuated by a factor of $1 / 10$ to $1 / 100$ with a combination of neutral density filters to avoid the pumping (optical quenching) effect of excited level populations by the probing laser itself. The transmitted intensity of the beam was detected through a monochromator by a photomultiplier with a fast current amplifier. The typical beam diameter was estimated to be about $10 \mu \mathrm{m}$ at the center of the gas gap in a discharge cell. The measurement was performed at $11 \times 7$ points in the $x-y$ plane of a discharge cell as shown in Fig. 1(a).

The temporal behavior of metastable Xe atoms generally has a long decay time constant. Therefore, in order to identify the zero absorption level, the applied voltage wave form was programmed to have a train of 6 pulses at each electrode followed by an off period of $30 \mu$ s. The absorption signal at the last pulse was used for the data analysis. The maximum absorption rates for the $1 s_{5}$ and $1 s_{4}$ levels were about $30 \%$ and $5 \%$, so the absorption signals were averaged by a digitizing oscilloscope over 5000 and 20000 times, respectively, to improve the signal-to-noise ratio.

\section{B. Absolute density derivation}

Absorption rate $A(\nu)$ of the incident laser intensity $I_{0}$ for an optical transition at frequency $\nu$ is given by

$$
A(\nu)=1-\frac{I}{I_{0}}=1-\exp [-k(\nu) l],
$$

where $I$ is the transmitted intensity, $l$ is the absorption length corresponding to the gas gap, and $k(\nu)$ is the absorption coefficient of which frequency dependence is given by the line profile of the transition. If the line profile has a single Gaussian shape given by the Doppler broadening, the density $N_{1}$ in the lower level is derived by

$$
N_{1}=\frac{\Delta \nu_{d}}{2}\left(\frac{\ln 2}{\pi}\right)^{-1 / 2} \frac{8 \pi g_{1}}{\lambda_{21}^{2} g_{2} A_{21}} k\left(\nu_{0}\right),
$$

where $k\left(\nu_{0}\right)$ is the absorption coefficient per unit length at the line center $\nu_{0}, \Delta \nu_{d}$ is the Doppler width, $\lambda_{12}$ is the wavelength, $A_{21}$ is the transition probability, and $g_{1}$ and $g_{2}$ are the statistical weights of the lower and upper levels of the transition. However, the line profile in the present case is very complicated due to the many isotopes contained in natural Xe gas, which produce isotope shifts ${ }^{11}$ and hyperfine structures. ${ }^{12}$ The effect of pressure broadening and shift of each component becomes more important at high filling pressures. ${ }^{9}$ Therefore, in order to derive the absolute densities of the excited $\mathrm{Xe}$ atoms from the absorption signal, we have to know the precise line profiles of the transitions used in the measurement.

For this purpose, the wavelength of the diode laser was scanned manually with the piezo driver. An example of the absorption profile of the $828.0 \mathrm{~nm}$ line is shown in Fig. 3. If 


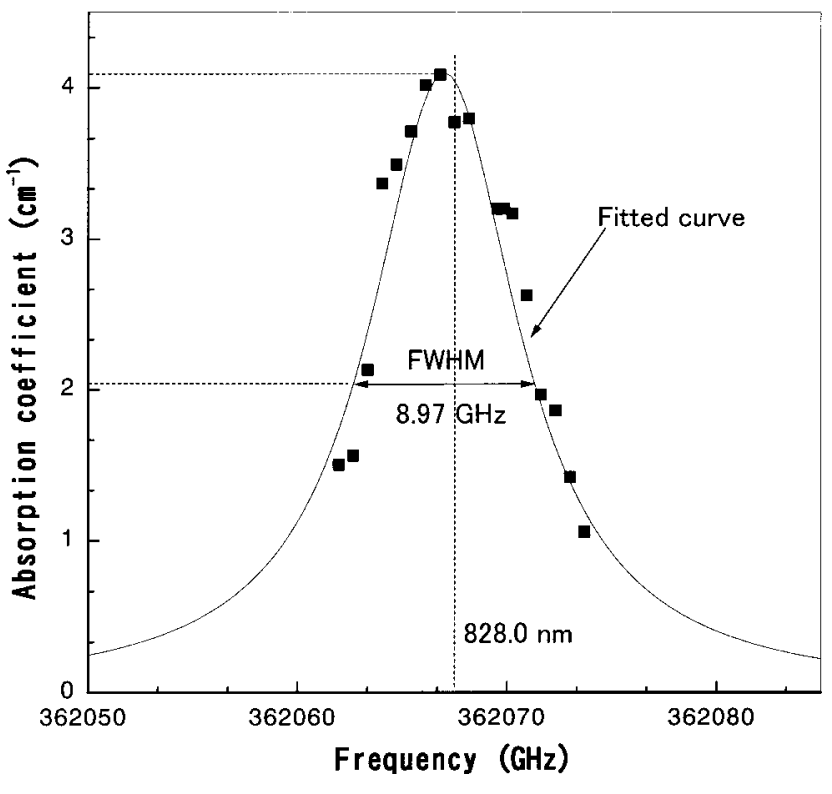

FIG. 3. Absorption line profile of $828.0 \mathrm{~nm}$ transition measured in a PDP panel containing $\mathrm{Ne}-\mathrm{Xe}(10 \%)$ mixture at 350 Torr. Measured data points are fitted by a Voigt profile from which integrated area is calculated.

we take the self-broadening coefficient to be $1.60 \times 10^{-19}$ $\mathrm{cm}^{-1} \mathrm{~cm}^{3}$ according to our previous measurements, ${ }^{13}$ the foreign-gas broadening coefficient by $\mathrm{Ne}$ is derived to be $0.94 \times 10^{-20} \mathrm{~cm}^{-1} \mathrm{~cm}^{3}$. Similarly, the coefficients for the $823.1 \mathrm{~nm}$ line are estimated to be $3.15 \times 10^{-20}$ and 1.37 $\times 10^{-20} \mathrm{~cm}^{-1} \mathrm{~cm}^{3}$, respectively. The slight shift that can be seen in Fig. 3 is mostly due to the pressure effect of Ne.

Due to the complicated structure and pressure broadening of the line profile, a correction factor $H$ must be multiplied to the right-hand side of Eq. (2) when the measured absorption coefficient at the fixed laser frequency, which has been tuned at the position shown by a dotted line in Fig. 3, is used as $k\left(\nu_{0}\right)$. Practically, since the effect of the line shift is small, the value of $H$ is given by the ratio of the peak height of the hypothetical single Doppler profile to that of the actual (measured) line profile after normalizing each line profile so that the integrated area becomes unity. In the present case, the correction factors for the 828.0 and $823.1 \mathrm{~nm}$ lines are evaluated to be 33.8 and 22.0 , respectively.

\section{RESULTS AND DISCUSSION}

\section{A. Spatiotemporal behaviors}

Measured results of the spatiotemporal behaviors of $\mathrm{Xe}$ atoms in the $1 s_{5}$ and $1 s_{4}$ states at $V_{s}=200 \mathrm{~V}$ are shown in Figs. 4 and 5, respectively, by three-dimensional plots in a series of time steps over a half cycle when the positive voltage pulse is applied to the electrode on the left-hand side. The $(11 \times 7)$ positions in the $x-y$ plane in Fig. 4 correspond to the assignment in Fig. 1(a). It can be seen that a sharp peak appears on the temporal anode (left-hand) side near the bus line, while a broader peak appears on the cathode side and is distributed over the entire electrode area. The maximum densities of the $1 s_{5}$ and $1 s_{4}$ levels at the temporal anode side are about $5 \times 10^{13}$ and $2 \times 10^{13} \mathrm{~cm}^{-3}$, respectively, while those values are $2 \times 10^{13}$ and $1 \times 10^{13} \mathrm{~cm}^{-3}$ on

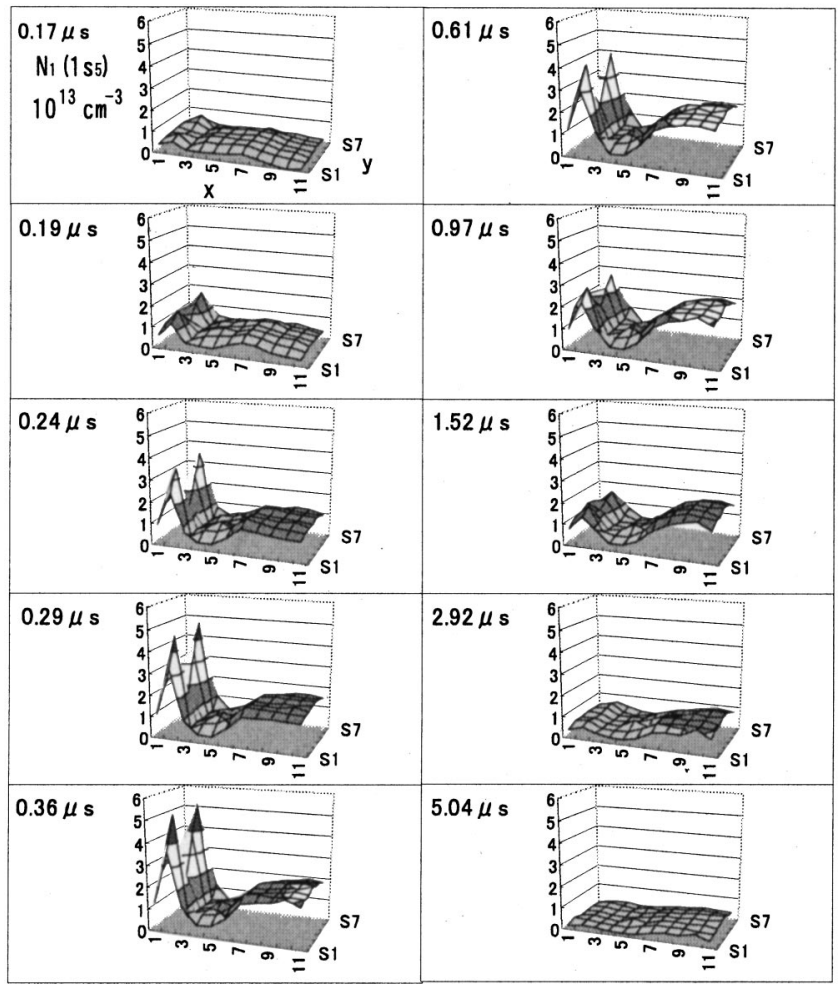

FIG. 4. Spatiotemporal behavior of $\mathrm{Xe}\left(1 s_{5}\right)$ atom density $N_{1}\left(1 s_{5}\right)$ in units of $10^{13} \mathrm{~cm}^{-3}$ measured in a half-cycle when the left-hand side is working as a temporal anode. Measured $x-y$ positions correspond to matrix shown in Fig. 1. Voltage pulse of $200 \mathrm{~V}$ peak and $2 \mu$ s duration is applied to the left-hand side electrode at $t=0$.

the temporal cathode side. The anode peak rises slightly faster than the cathode peak as can be seen more clearly in Fig. 7.

A doubly peaked or centrally bent shape in $y$ direction can be noticed on the anode peak in Figs. 4 and 5. In order to show the shape more clearly, a contour diagram is shown in Fig. 6 for the spatial profile of $\mathrm{Xe}\left(1 s_{5}\right)$ density at a time of $0.5 \mu \mathrm{s}$. The dip in the central part of the anode peak is about $40 \%$ of the maximum in Fig. 6(b). The bent shape, however, may not be inherent to the coplanar-type discharge cell. In fact, at the beginning of our experiment, only one peak used to appear as shown in Fig. 6(a). After a certain operation time of several tens of hours, it has split irreversibly into two peaks, probably due to a change in the electrode surface condition. At the moment, we have no confidence whether the charging effect on the insulating ribs or any other cause can produce the doubly peaked shape of the anode peak.

In order to see the behavior at the early period of the discharge in more detail, the spatiotemporal behavior of $\mathrm{Xe}\left(1 s_{5}\right)$ is shown in Fig. 7 in a contour diagram. The diagram shows that a slightly larger density remains on the anode side, which has worked as a temporal cathode in the preceding half-cycle, and the density starts to rise at the anode side on the inner edge of the electrode gap. Then, a large peak rises at the edge of the anode bus line. At the same time, the peak at the gap edge starts to extend to the cathode side toward the bus line. In order to examine how the 


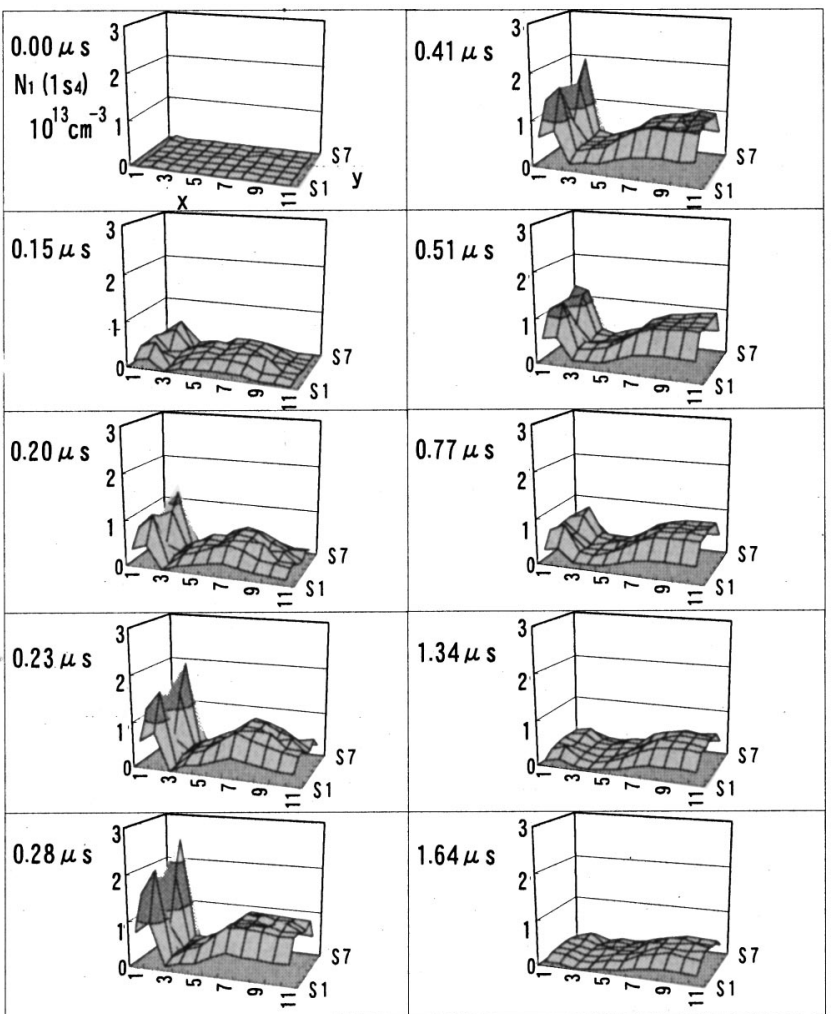

FIG. 5. Spatiotemporal behavior of $\mathrm{Xe}\left(1 s_{4}\right)$ atom density $N_{1}\left(1 s_{4}\right)$ in units of $10^{13} \mathrm{~cm}^{-3}$ measured in a half-cycle when the left-hand side is working as a temporal anode. Measured $x-y$ positions and conditions are the same as in Fig. 4.

residual density of excited atoms affects the successive discharges, the period of square wave pulses was changed from 12 to $18 \mu \mathrm{s}$. The delay time of the discharge became longer from 160 to $300 \mathrm{~ns}$ as the period was increased, while the

(a)

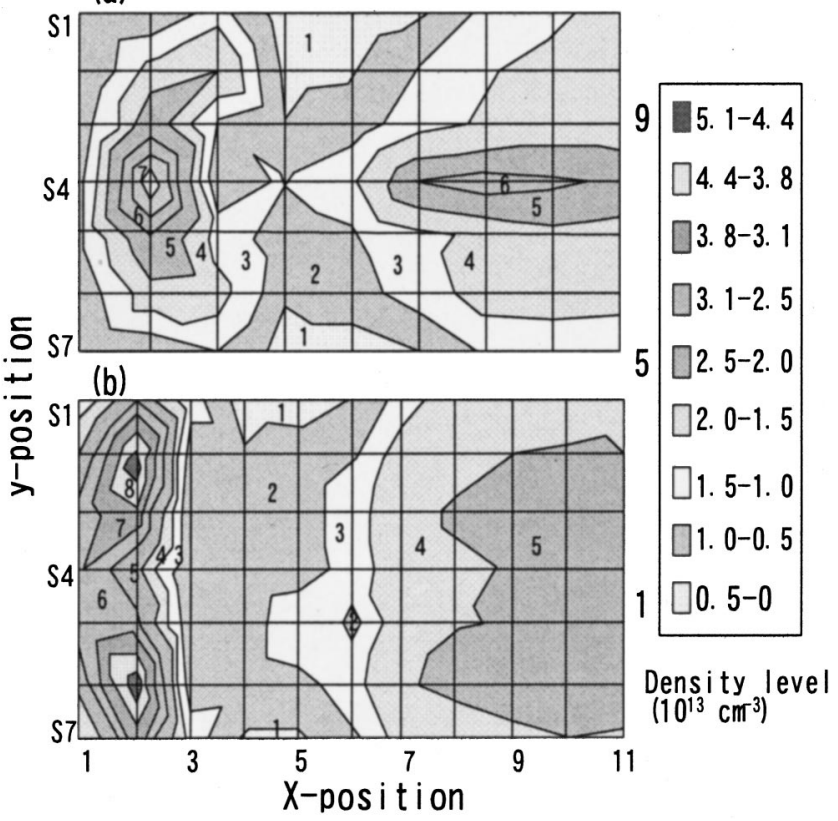

FIG. 6. Contour diagram of spatial profile of $\mathrm{Xe}\left(1 s_{5}\right)$ density at $t$ $=0.5 \mu$ s measured (a) at beginning of experiment and (b) after seasoning.

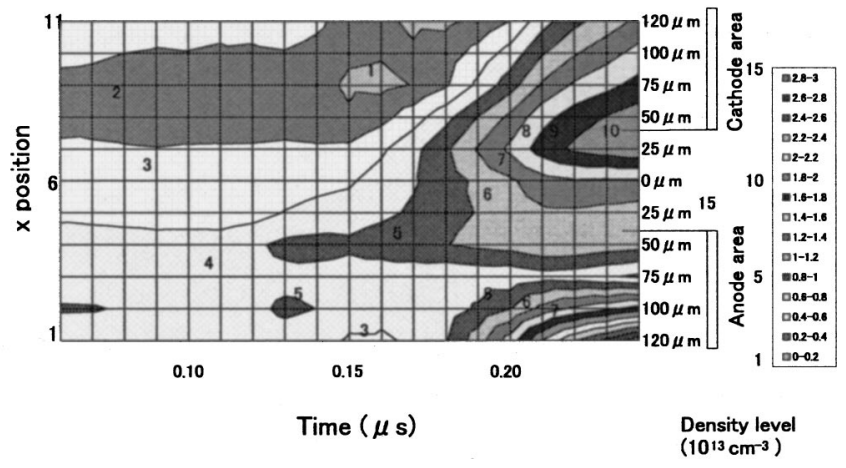

FIG. 7. Contour diagram of spatiotemporal behavior of $\mathrm{Xe}\left(1 s_{5}\right)$ atom density measured at early phase of discharge when voltage pulse of $200 \mathrm{~V}$ peak and $2 \mu$ s duration is applied to the lower side electrode (temporal anode).

heights of the anode and cathode peaks did not change noticeably.

A logarithmic plot of the $\mathrm{Xe}\left(1 s_{5}\right)$ atom density against time is shown in Fig. 8 over two half-cycles where the measured side is working as the temporal anode and cathode. The measured position was $(2, \mathrm{~S} 6)$. The density does not decrease immediately after the end of the current pulse when the measured side works as the temporal cathode. This suggests that there are some production processes in the early afterglow, probably due to the dissociative recombination of $\mathrm{Xe}_{2}^{+}$ions followed by radiation cascades to $1 s$ levels. On the contrary, the density on the temporal anode side decays faster in the early afterglow, indicating the presence of some loss processes. The stepwise excitation and ionization by electron collisions are possible mechanisms of the fast decay. The diffusion in higher modes also plays a role in enhancing the decay rate because the distribution of excited Xe atoms is concentrated in a narrow region near the bus line on the anode side. The time constant of the decay in late afterglow, however, takes the same value of $2.5 \mu \mathrm{s}$ on both sides, which agrees with the value of $2.6 \mu$ s given by the inverse of the three-body loss rate of $\mathrm{Xe}\left(1 s_{5}\right)$ atoms as described below. This means that the diffusional loss rate of $\mathrm{Xe}\left(1 s_{5}\right)$ atoms is negligible in the present case.

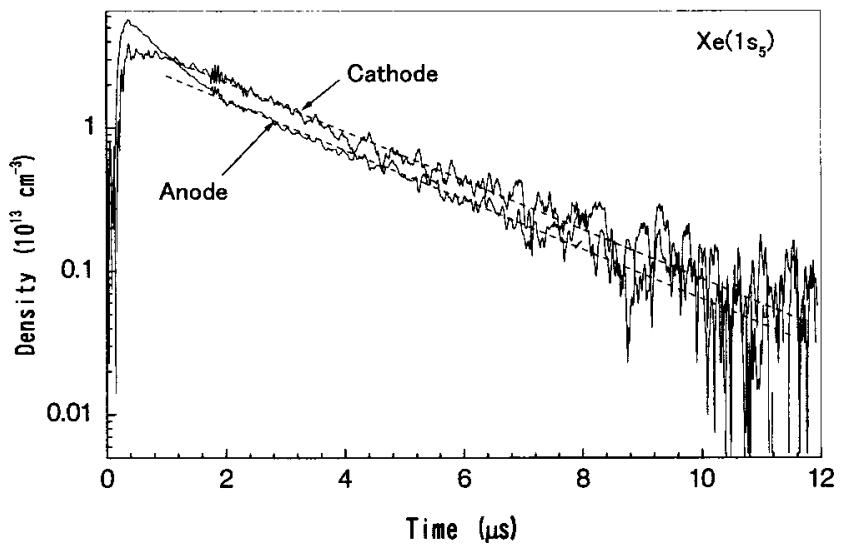

FIG. 8. Logarithmic plot of $\mathrm{Xe}\left(1 s_{5}\right)$ atom density against time measured at a position $(2, \mathrm{~S} 6)$ in two-half cycles when measured point is working as temporal anode and cathode. Dashed lines are fitted to decay in late afterglow. 


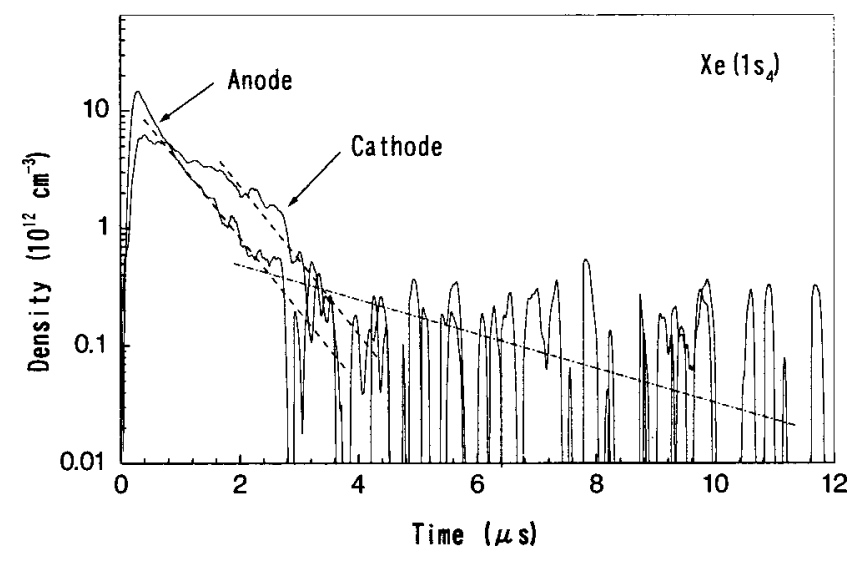

FIG. 9. Logarithmic plot of $\mathrm{Xe}\left(1 s_{4}\right)$ atom density against time measured as in Fig. 7. Dashed lines are fitted to decay from 1 to $3 \mu \mathrm{s}$, and dot-dashed line with $2.5 \mu$ s time constant is drawn for reference to show behavior in late afterglow.

The decay behavior of $\mathrm{Xe}\left(1 s_{4}\right)$ atoms is shown in Fig. 9. The characteristic behaviors on the cathode and anode sides at the beginning of afterglow are similar to the case of $\mathrm{Xe}\left(1 s_{5}\right)$ atoms; the decay on the cathode side is not single exponential but has a swell in the early afterglow. The decay time constant on the anode side is estimated to be $0.37 \mu \mathrm{s}$ from the slope of a fitted line in Fig. 9, which is in good agreement with the effective lifetime of about $0.34 \mu$ s calculated from the rates of imprisoned resonance radiation and three-body collision (see Sec. IIIC). The figure also shows that a slow decay component remains in the late afterglow. This may be due to the collisional population transfer from the $1 s_{5}$ level to the $1 s_{4}$ level. (A dot-dashed line with the same time constant of $2.5 \mu \mathrm{s}$ as in the metastable atom decay is shown in Fig. 9 as a reference.)

\section{B. Current and visible-emission wave forms}

The current wave form was measured by a digital oscilloscope using a current probe of $100 \mathrm{MHz}$ bandwidth. One line containing 640 cells was lit at the same time to increase the total current. The displacement current was subtracted from the measured wave form using the current wave form without discharge operated slightly below the ignition voltage. The wave form so processed is shown in Fig. 10. It has a half-width of about 70 ns. The average current can be obtained by integrating the current wave form over $6 \mu \mathrm{s}$, which is used in the estimation of efficiency described below. The average current estimated in this way was 4.7 to 5.5 $\mu \mathrm{A}$, depending on the operation condition. This amounts to about $60 \%$ of the mean current per cell measured by the external total current flowing into the driving circuit but is a reasonable level if the circuit loss is taken into account.

The emission from $\mathrm{Ne}$ atoms in the red spectral range was also measured. In this measurement, the test panel was faced directly to the entrance slit of a monochromator. A spectral line at $640.2 \mathrm{~nm}\left(2 p_{9}-1 s_{5}\right)$ was chosen due to its having the strongest intensity among many visible lines. The experimentally observed wave form was delayed from the current pulse by about $60 \mathrm{~ns}$, which was caused by the propagation delays through the measuring electronics sys-

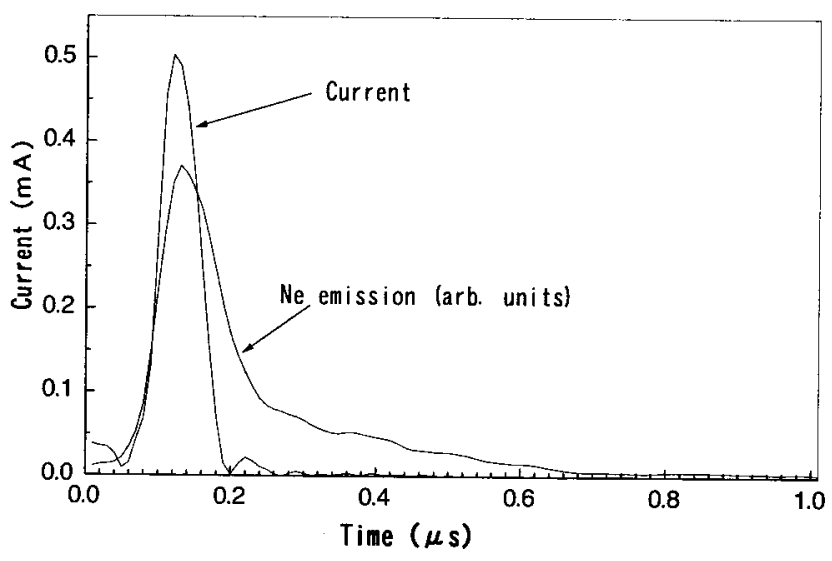

FIG. 10. Wave forms of discharge current measured by current probe and $\mathrm{Ne}$ visible emission at $640.2 \mathrm{~nm}$.

tem. The wave form corrected for the delay is superposed in Fig. 10. (The same correction has been done on all temporal behaviors shown in this article.) The wave form has a halfwidth of about $90 \mathrm{~ns}$, which is slightly larger than that of the current wave form due to the finite lifetime of the radiative transition of $23 \mathrm{~ns}$. The slower decay in the tail may include the contribution of the radiative cascade from upper levels via a dissociative recombination of $\mathrm{Xe}_{2}^{+}$. At any rate, it suggests that the emission wave form can work as a good monitor of the current if the effect of the finite lifetime is deconvoluted from the wave form.

\section{Estimation of luminous efficiency}

The absolute emission intensity of the $147 \mathrm{~nm}$ atomic line can be derived from the measured spatiotemporal density of $\mathrm{Xe}\left(1 s_{4}\right)$ atoms. The measured value, however, gives the average density $\bar{N}_{1}$ over the absorption length through the gas gap. Therefore, it is simply assumed here that the 147 $\mathrm{nm}$ line is emitted with an effective transition probability for the imprisoned radiation. ${ }^{14}$ From the transition probability $A_{10}$ and the wavelength $\lambda_{10}$ of $147 \mathrm{~nm}$ line, the effective value $A_{10}^{\prime}$ is given by

$$
A_{10}^{\prime}=\frac{B}{\sqrt{3 \pi^{2} l / \lambda_{10}}} A_{10} \equiv g A_{10},
$$

where $B$ is a constant, $l$ is the gas gap (taken as the minimum length in $x, y$, and $z$ directions), and $g$ is defined as the escape factor. If a literature value of 1.15 is taken for $B,{ }^{14}$ the value of $g$ is estimated to be $7.1 \times 10^{-3}$. Accordingly, $A_{10}^{\prime}$ becomes $2.0 \times 10^{6} \mathrm{~s}^{-1}$, if a reported value of $2.8 \times 10^{8} \mathrm{~s}^{-1}$ is taken for $A_{10} \cdot{ }^{15}$ Thus, the radiation power at $147 \mathrm{~nm}$ is calculated from

$$
\begin{aligned}
P_{147}= & h \nu_{10} A_{10}^{\prime} \iiint_{V} N_{1}\left(1 s_{4}\right) d x d y d z=2.7 \\
& \times 10^{-12} l \iint_{S} \bar{N}_{1}\left(1 s_{4}\right) d x d y
\end{aligned}
$$




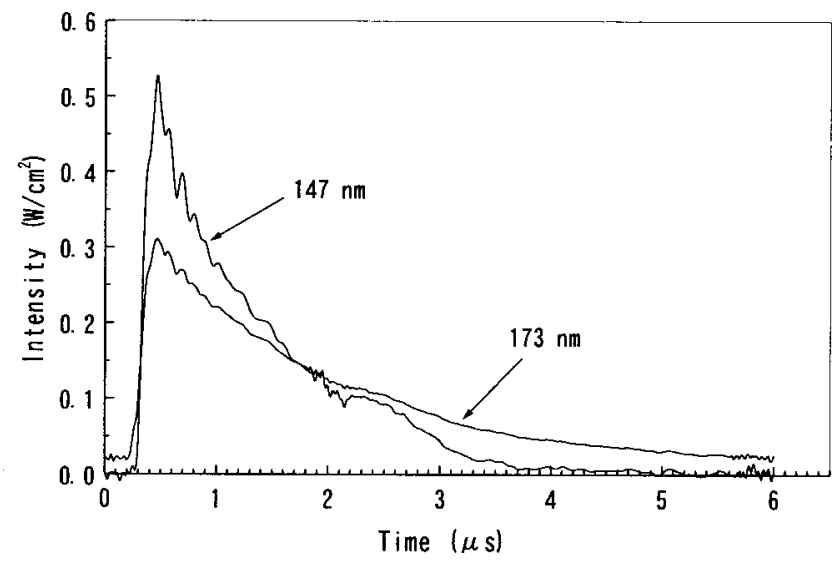

FIG. 11. Spatially integrated temporal behaviors of VUV emissions at 147 and $173 \mathrm{~nm}$ estimated from measured spatiotemporal $\mathrm{Xe}\left(1 s_{4}\right)$ and $\mathrm{Xe}\left(1 s_{5}\right)$ atom densities.

where $h \nu_{10}$ is the photon energy at $147 \mathrm{~nm}, V$ is the volume of a discharge cell, and $S$ is the total area in the $x-y$ plane.

The absolute intensity of the $173 \mathrm{~nm}$ band from $\mathrm{Xe}_{2}^{*}$ dimmers can also be derived from the measured density of $\mathrm{Xe}\left(1 s_{5}\right)$ atoms, assuming that the three-body collisions are the rate-determining process for the dimmer formation:

$$
\begin{aligned}
& \mathrm{Xe}\left(1 s_{5}\right)+2 \mathrm{Xe} \rightarrow \mathrm{Xe}_{2}\left(1_{u}, 0_{u}^{-}\right), \\
& \mathrm{Xe}\left(1 s_{5}\right)+\mathrm{Xe}+\mathrm{Ne} \rightarrow \mathrm{Xe}_{2}\left(1_{u}, 0_{u}^{-}\right) .
\end{aligned}
$$

The reported rate coefficients for these reactions are 8.53 $\times 10^{-32}$ and $4.07 \times 10^{-32} \mathrm{~cm}^{6} \mathrm{~s}^{-1}$, respectively. ${ }^{16}$ Therefore, the total rate for the formation of $\mathrm{Xe}_{2}\left(1_{u}, 0_{u}^{-}\right)$excimers becomes $3.83 \times 10^{5} \mathrm{~s}^{-1}$ at a total pressure of 350 Torr with $10 \% \mathrm{Xe}$ in Ne. Some part of the above three-body processes may produce $\mathrm{Xe}_{2}\left(0_{u}^{+}\right)$at higher vibrational levels, which lead to the $152 \mathrm{~nm}$ band emission. ${ }^{17}$ However, from the observed spectra in the VUV region in usual cases, ${ }^{18}$ the wavelength-integrated intensity of the $152 \mathrm{~nm}$ band is estimated to be less than $10 \%$ to $20 \%$ of the intensity of the 173 $\mathrm{nm}$ band. There included also is the contribution from $\mathrm{Xe}\left(1 s_{4}\right)$ atoms to the $152 \mathrm{~nm}$ band emission as described below. Therefore, we assume here that the three-body processes yield predominantly $\mathrm{Xe}_{2}\left(1_{u}, 0_{u}^{-}\right)$, of which the population relax to lower vibrational levels, leading to the $173 \mathrm{~nm}$ band emission. From $\mathrm{Xe}\left(1 s_{4}\right)$ atoms, $\mathrm{Xe}_{2}\left(0_{u}^{+}\right)$excimers are formed through reactions similar to Eqs. (5a) and (5b). The estimated total rate becomes $9.67 \times 10^{5} \mathrm{~s}^{-1}$ when we apply the reported rate constants, ${ }^{16}$ which corresponds to about $1 / 3$ of the rate for the $147 \mathrm{~nm}$ emission. The emission from higher lying vibrational levels of $\mathrm{Xe}_{2}\left(\mathrm{O}_{u}^{+}\right)$occurs at around $152 \mathrm{~nm}$. This estimation, however, may be the upper limit since the rate constant $1.55 \times 10^{-31} \mathrm{~cm}^{6} \mathrm{~s}^{-1}$ used for the $\mathrm{Xe}\left(1 s_{4}\right)+2 \mathrm{Xe}$ process $^{16}$ is large. In the following, we simply include this contribution into the intensity of the $147 \mathrm{~nm}$ emission since the band appears at its red wing, although the relaxation to lower vibrational levels and the collisional transfer from $\mathrm{Xe}_{2}\left(0_{u}^{+}\right)$to $\mathrm{Xe}_{2}\left(1_{u}\right)$ may convert some part of the intensity into the $173 \mathrm{~nm}$ band emission.

Temporal behaviors of the $147 \mathrm{~nm}$ line and $173 \mathrm{~nm}$ band emissions so estimated are shown in Fig. 11, where the spa-

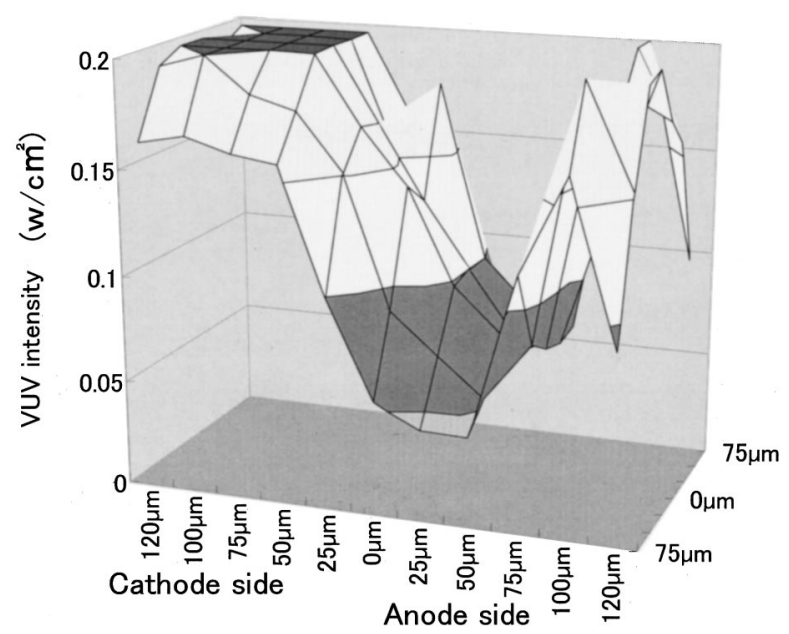

FIG. 12. Spatial distribution of total VUV emission integrated over a halfcycle when the right-hand side is working as a temporal anode.

tial distribution is integrated over the entire area of a discharge cell. The $147 \mathrm{~nm}$ emission rises faster and decays faster in contrast to the $173 \mathrm{~nm}$ emission. However, their temporally integrated values become comparable in our measurement at the Xe concentration of $10 \%$. It is expected that the contribution of the $173 \mathrm{~nm}$ emission becomes larger as the Xe concentration increases because the rate of the threebody processes for the production of $\mathrm{Xe}_{2}^{*}$ increases rapidly with the Xe concentration.

The spatial distribution of the total VUV emission (sum of the 147 and $173 \mathrm{~nm}$ emissions) is temporally integrated over a half-cycle as shown in Fig. 12, where the left-hand side worked as the temporal cathode. The estimated emission from the cathode area amounts to $65 \%$ and that from the anode side is about $35 \%$. This means that the contribution from the anode side is not negligible. This is a significant feature in an ac-type cell, as noted in the comparison with a dc-type cell below.

The luminosity and total efficiency of the VUV emission estimated in this way are plotted in Fig. 13 as a function of the average current measured by changing the applied pulse voltage from 190 to $210 \mathrm{~V}$. The luminosity increases slightly, but the efficiency decreases as the power input increases. This tendency is consistent with reported simulation results. ${ }^{5-7} \mathrm{~A}$ possible reason may be attributed to the electron quenching of excited levels by stepwise excitation and cumulative ionization.

\section{Difference between ac and dc types}

The behaviors of the excited Xe atom densities measured in an ac-type cell are compared with the results obtained previously in a dc-type cell. ${ }^{19}$ It is interesting that the peak values of the $\mathrm{Xe}\left(1 s_{4}\right)$ and $\mathrm{Xe}\left(1 s_{5}\right)$ atoms are comparable in the order of $10^{13}-10^{14} \mathrm{~cm}^{-3}$ in both cases. As for spatial distribution, however, a sharp peak in front of the cathode (negative glow) is predominant in the dc-type cell, and there appeared a smaller and broader peak (positive column) toward the anode as the electrode gap increased. Therefore, in a dc-type cell the contribution from the negative glow pre- 

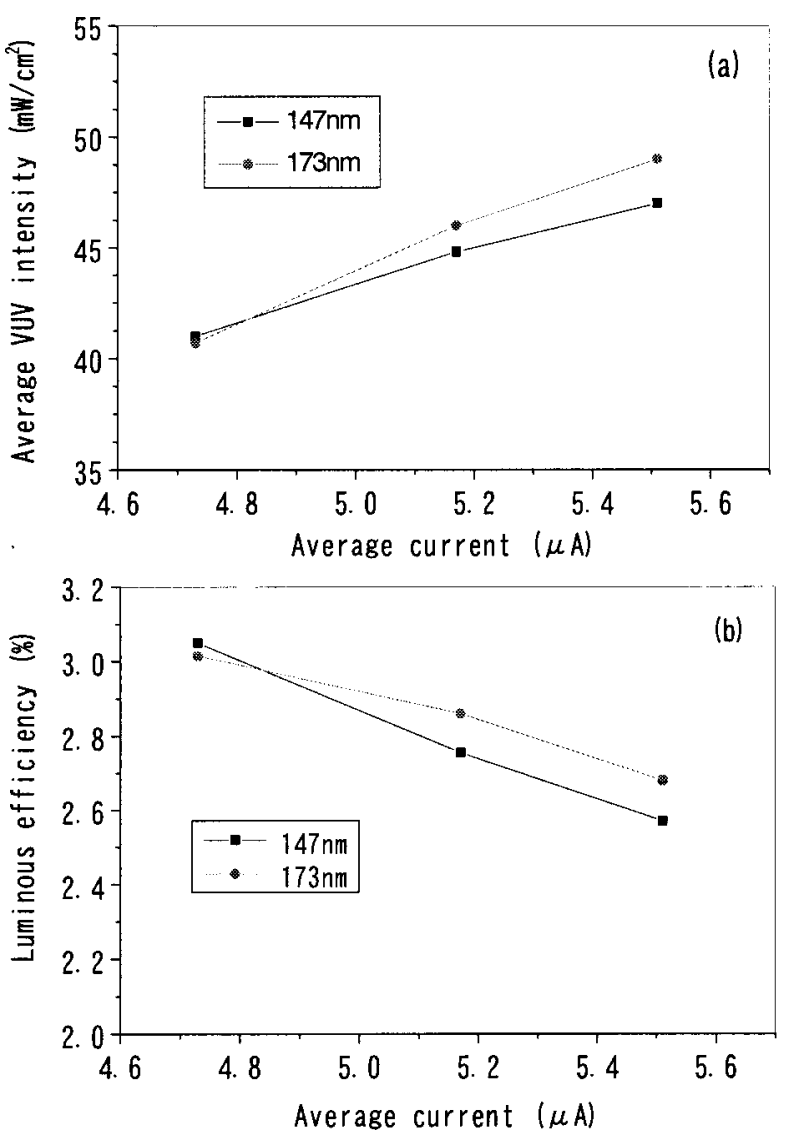

FIG. 13. Estimated luminosity and luminous efficiency of VUV emissions at 147 and $173 \mathrm{~nm}$ plotted as a function of average current.

dominates in the total VUV emission. On the contrary, in an ac-type cell the contribution from the anode side amounts to more than $30 \%$ as mentioned previously. In a dc-type cell the current keeps flowing during applied pulse width on the order of $1 \mu \mathrm{s}$. However, in the quasisteady state after the cathode fall is formed in the early phase within a few hundreds $\mathrm{ns}$, the electric field on the anode side becomes much smaller, and the energy of electrons decreases. Thus, there is no effective production of excited atoms in the region from the negative glow to the anode. In contrast to this feature, in an ac-type cell both emissions on the cathode and anode sides reach their maximum values soon after the cathode fall is formed and start to decay with the rapid decrease in the current. This behavior produces the relatively large contribution from the anode side. More quantitative comparisons will be done in a following paper on the characterization of a dc-type PDP.

\section{CONCLUDING REMARKS}

The spatiotemporal behaviors of the excited Xe atom densities in the $1 s_{4}$ and $1 s_{5}$ levels were successfully measured, for the first time, in a realistic cell of an ac-type PDP. The maximum values of $\mathrm{Xe}\left(1 s_{4}\right)$ and $\mathrm{Xe}\left(1 s_{5}\right)$ atom densities were about $2 \times 10^{13}$ and $5 \times 10^{13} \mathrm{~cm}^{-3}$, respectively. This type of measurement, however, gives inherently averaged values along the gas gap ( $z$ direction). If the density along the $z$ axis is given by a cosine distribution, the peak values become $3 \times 10^{13}$ and $8 \times 10^{13} \mathrm{~cm}^{-3}$, respectively. It is worthwhile to compare these values with the estimated values close to $10^{14} \mathrm{~cm}^{-3}$ by simulation, ${ }^{5}$ although the conditions in both cases are not completely equivalent.

The decay of $\mathrm{Xe}\left(1 s_{5}\right)$ atoms was governed by the threebody collision rate to form $\mathrm{Xe}_{2}^{*}$ excimers, while the decay of $\mathrm{Xe}\left(1 s_{4}\right)$ atoms was predominantly determined by the effective lifetime of imprisoned resonance radiation. It was ascertained that the residual metastable atoms play a role in shortening the delay time of the discharge.

From the measured results, the absolute values of the VUV emission intensities at the $147 \mu \mathrm{m}$ atomic line and the $173 \mu \mathrm{m}$ excimer band were estimated. The absolute value of luminous efficiency of $5 \%$ to $6 \%$ at a gas pressure of 350 Torr with a Xe concentration of $10 \%$ is smaller but consistent with the simulated values of $11 \%$ at 560 Torr (in a one-dimensional model) ${ }^{5}$ and $15 \%$ at 450 Torr (in a twodimensional model $)^{20}$, if the pressure effect is taken into account. The total VUV emission integrated over a half-cycle from the temporal cathode region predominates over that from the anode region, but the ratio is about 7:3 and thus the contribution from the anode side is not negligible.

The intensity of the VUV emission tends to saturate and the efficiency decreases as the mean current increases. This is attributed to the electron quenching of excited Xe atoms by stepwise excitation and/or ionization processes. An effective method to reduce this loss rate is to increase the total pressure or the Xe concentration, although this would cause an increase in the ignition and sustain voltages. ${ }^{5,21}$ In order to minimize this voltage increase effect, the development of materials with higher efficiency of the secondary electron emission ( $\gamma$ effect) seems essential to improvement in luminous efficiency. ${ }^{22}$

In the present work, a two-dimensional measurement in the $x-y$ plane was performed on excited Xe atom densities (front view). However, since most of the existing simulations are done in the $x-z$ plane, the side (cross sectional) view measurement is also required. We have started a preliminary work on such a measurement by using a specially designed cell for the purpose. ${ }^{23}$ By combining these two measurements from both sides, we can obtain a three-dimensional image of the dynamic behaviors of excited atoms in a realistic PDP cell. The results will be compared with those of other three-dimensional simulations conducted in recent years. ${ }^{24,25}$

\section{ACKNOWLEDGMENTS}

The authors are grateful to T. Tanaka and K. Ito, who had been working at Hyundai Electronics Japan, for preparing the experimental setup. This work is supported in part by a Grant-in-Aids from the Japanese Ministry of Education, Science, Sports and Culture.

\footnotetext{
${ }^{1}$ See, e.g., T. Shinoda, J. Plasma Fusion Res. 74, 109 (1998) (in Japanese); T. Hirosde and K. Kariya, Proceedings of the Sixth International Display Workshop (IDW '99), Sendai (Society of Information Display, Tokyo, 1999), p. 11.

${ }^{2}$ T. Shinoda, M. Wakitani, T. Nanto, K. Yoshikawa, A. Otsuka, and T.
} 
Hirose, SID '93 Digest (Society of Information Display, Playa del Rey, CA, 1993), p. 161.

${ }^{3}$ M. Sawa, H. Uchiike, S. Zhang, and K. Yoshida, SID '98 Digest (Society of Information Display, Playa del Rey, CA, 1998), p. 361.

${ }^{4}$ H. Seob, J.-H. Seo, C.-K. Yoon, J.-K. Kim, and K.-W. Whang, J. Appl. Phys. 85, 3092 (1999).

${ }^{5}$ J. Meunier, Ph. Belenguer, and J.-P. Boeuf, J. Appl. Phys. 78, 731 (1995).

${ }^{6}$ C. Punset, J.-P. Boeuf, and L. C. Pitchford, J. Appl. Phys. 83, 1884 (1998).

${ }^{7}$ S. Rauf and M. J. Kushner, J. Appl. Phys. 85, 3460 (1999).

${ }^{8}$ Y. Ikeda, J. P. Verboncoeur, P. J. Christenson, and C. K. Birdsall, J. Appl. Phys. 86, 2431 (1999).

${ }^{9}$ K. Tachibana, N. Kosigi, and T. Sakai, Appl. Phys. Lett. 65, 935 (1994).

${ }^{10}$ T. Tanaka, K. Itoh, H. Nagasaka, T. Sakai, S. Feng, and K. Tachibana, Technical Report No. EID98-24, The Institute of Electronics, Information and Communication Engineers, Tokyo, 1998, p. 13 (in Japanese).

${ }^{11}$ D. A. Jackson and M.C. Coulombe, Proc. R. Soc. London, Ser. A 338, 277 (1974).

${ }^{12}$ D. A. Jackson and M. C. Coulombe, Proc. R. Soc. London, Ser. A 327, 137 (1972); 335, 127 (1973).

${ }^{13}$ N. Kitagawa, Master thesis, Kyoto Institute of Technology, 1989.

${ }^{14}$ T. Holstein, Phys. Rev. 72, 1212 (1947); 83, 1159 (1951).

${ }^{15}$ W. L. Wiese and G. A. Martin, Wavelengths and Transition Probabilities for Atoms and Atomic Ions, NSRDS-NBS 68, Part II (U.S. GPO, Washington, DC, 1980), p. 405.

${ }^{16}$ J. Galy, K. Aouane, A. Birot, H. Brunet, and P. Millet, J. Phys. B 26, 447 (1993).

${ }^{17}$ H. D. Wenck, S. S. Hasnain, M. M. Nikitin, K. Sommer, and G. Zimmerer, Chem. Phys. Lett. 66, 138 (1979).

${ }^{18}$ T. Tamida, S. J. Sanders, and M. Tanaka, Jpn. J. Appl. Phys., Part 1 39, 583 (2000).

${ }^{19}$ N. Kosugi, K. Tachibana, M. Seki, and T. Sakai, Proceedings of the Sixth International Display Workshop (IDW '99), Sendai (Soc. Information Display, Tokyo, 1999), p. 687.

${ }^{20}$ M. Klein, R. Snjkers, and H. Hagelaar, Proceedings of the Sixth International Display Workshop (IDW '99), Sendai (Society of Information Display, Tokyo, 1999), p. 695.

${ }^{21}$ S. Hashiguch and K. Tachibana, Trans. Inst. Electr. Eng. Jpn., Part A 120, 532 (2000) (in Japanese).

${ }^{22}$ K. S. Moon, J. Lee, and K.-W. Whang, J. Appl. Phys. 86, 4049 (1999), and references therein.

${ }^{23}$ F. Katafuchi, K. Tachibana, and T. Sakai, in Ref. 19, p. 1115.

${ }^{24}$ P. J. Drallos, V. N. Khundik, and V. P. Nagorny, SID '98 Digest (Society of Information Display, Playa del Rey, CA, 1998), p. 632.

${ }^{25}$ H.-S. Jeong, Y. Murakami, and M. Seki, in Ref. 19, p. 671. 